Analysis 20,1 - $13(2000)$

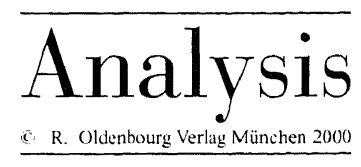

\title{
ASYMPTOTIC ESTIMATES FOR GENERALIZED STIRLING NUMBERS
}

R. Chelluri, L.B. Richmond and N.M. Temme

Received: May 11, 1999

Abstract. Uniform asymptotic expansions are given for the Stirling numbers of the first kind for integral arguments and for the second kind as defined for real arguments by Flajolet and Prodinger. The logconcavity of the resulting real valued function of Flajolet and Prodinger is established for a range including the classical integral domain.

Subject classification (A.MS 1991) 11B73, 41A60

\section{Introduction}

Recently Flajolet and Prodinger [4] have given a solution to the problem of Graham, Knuth and Patashnik [5] which asks for a good generalization of the Stirling numbers of the second kind, denoted here by $S_{k}^{n}$ for complex numbers $n$ and $k$. They define (equation (2) of [4])

$$
S_{x}^{y}=\frac{y ! 1}{x ! 2 \pi i} \int_{C} z^{-y-1}\left(e^{z}-1\right)^{x} d z
$$

Here $C$ is a Hankel contour which starts at $-\infty$, circles the origin and goes back to $-\infty$ subject to $|\operatorname{Im} z|<2 \pi$. Flajolet and Prodinger do not consider this problem for Stirling numbers of the first kind in the same detail although they do establish the identity $S_{k}^{n}=s_{-n}^{-k}$, 
$s_{k}^{n}$ denotes the Stirling number of the first kind using their definition of $S_{x}^{y}$. The definition of $s_{x}^{y}$ they give is

$$
\left|s_{x}^{y}\right|=\frac{y !}{x !} \frac{1}{2 \pi i} \int_{C_{1}} \log ^{x}\left(\frac{1}{1-w}\right) \frac{d w}{w^{y+1}},
$$

where $C_{1}$ is the "raindrop contour", the image of $C$ under $w=e^{z}-1$. It is natural to consider the absolute value of the Stirling numbers of the first kind as Moser-Wyman [7] and Temme [11] have done since questions of sign are avoided and $\left|s_{k}^{n}\right|$ is the number of permutations on $n$ symbols with $k$ cycles. It is natural for us, following these authors, to define for positive real $x$ and $y$

$$
s_{x}^{y}=\frac{1}{2 \pi i} \int_{C_{2}} \frac{\Gamma(u+y+1)}{\Gamma(u+1)} \frac{d u}{u^{x+1}} .
$$

Here $C_{2}$ is any Jordan curve which circles the origin in the counterclockwise sense. Note that $\Gamma(u+y+1) / \Gamma(u+1)$ is a polynomial in $u$ for integral $y$ and has no singularities for positive nonintegral $y$ for $|u|<y$. We adopt this last definition, however we are primarily interested in positive integral values where the definitions agree.

Flajolet and Prodinger show that $S_{x}^{y}$ is for fixed $y$ an entire function of $x$ and for fixed $x$ a meromorphic function of $y$ with poles at the negative integers.

In this note we derive asymptotic estimates for these generalized Stirling numbers. Our estimates are completely analogous to the estimates stated by Temme [11] for the standard Stirling numbers and are uniform in $\delta<x \leq y, \delta$ a positive constant as $y \rightarrow \infty$ for the Stirling numbers of the second kind and uniform and for the first kind are uniform for integral $x$ and $y$ in the same ranges. One reason for doing this is that certain steps in the proof of Temme's results were omitted (see Odlyzko's comments [8] regarding this), another is to verify that these results hold for Flajolet and Prodinger's generalization. We derive Temme's results using the analysis of Moser and Wyman [6,7] which turns out to be easily adapted to the generalized numbers. We also, using the method of Merlini-Richmond [10] (based on work of Gardy [3]) show that $S_{x}^{y}$ is a logconcave function of $x$ for large $y$ and $\delta<x \leq y$.

We prove three theorems (we let $\delta$ denote a positive constant).

Let $u_{0}$ be the unique real positive solution of the equation

$$
\frac{m}{n} u_{0}=1-e^{-u_{0}}
$$

(It is easily seen that $u /\left(1-e^{-u}\right)$ is an increasing function). Let

$$
t_{0}=(y-x) / x, \quad \phi(u)=-y \log u+x \log \left(e^{u}-1\right),
$$


and

$$
A=\phi\left(u_{0}\right)-x t_{0}+(y-x) \log t_{0}
$$

Let

$$
f\left(t_{0}\right)=\left(t_{0} /\left(1+t_{0}\right)\left(u_{0}-t_{0}\right)\right)^{1 / 2}
$$

(We define $t_{0}, A$ and $f\left(t_{0}\right)$ as Temme [11] does). Finally let

$$
2 H_{0}(u)=e^{u}\left(e^{u}-1\right)^{-1}-u e^{u}\left(e^{u}-1\right)^{-2} .
$$

THEOREM 1. The relation

$$
S_{x}^{y} \sim e^{A} x^{y-x} f\left(t_{0}\right)\left(\begin{array}{l}
y \\
x
\end{array}\right),
$$

where $\left(\begin{array}{l}y \\ x\end{array}\right)=y ! / x !(y-x)$ ! holds uniformly as $y \rightarrow \infty$ for $\delta<x \leq y$. Furthermore if $\delta<x \leq y-y^{1 / 3}$ then

$$
S_{x}^{y}=\frac{y !\left(e^{u_{0}}-1\right)^{x}}{x !} \frac{1}{2 u_{0}^{y}}\left[\pi u_{0} x H_{0}\left(u_{0}\right)\right]^{1 / 2}\left[1+\mathcal{O}\left(y^{-1}\right)\right]
$$

where the $\mathcal{O}$-constant depends only on $\delta$. Finally, if $y-y^{1 / 3} \leq x \leq y$ then

$$
S_{x}^{y}=\frac{1}{2^{y-x}} \frac{y^{2(y-x)}}{(y-x) !}\left[1+\mathcal{O}\left(y^{-1 / 3}\right)\right]
$$

where the $\mathcal{O}$-constant is independent of $x$.

Now let $\psi(u)=\log [(u+1)(u+2) \cdots(u+[y])]-x \log u$. Let $u_{1}$ be the unique positive solution of $\psi^{\prime}(u)=0$ (see Temme [11] for a proof that $u_{1}$ is unique). Let $t_{1}=x /(y-x)$ and $B=\psi\left(u_{1}\right)-y \log \left(1+t_{1}\right)+x \log t_{1}$. Finally, let $g\left(t_{1}\right)=u_{1}^{-1}\left[x(y-x) / y \psi^{\prime \prime}\left(u_{1}\right)\right]^{1 / 2}$ and let $H=x-\sum_{h=0}^{\{y \mid-1} u_{1}^{2} /\left(u_{1}+h\right)^{2}$.

THEOREM 2. The relation

$$
s_{x}^{y} \sim e^{B} g\left(t_{1}\right)\left(\begin{array}{l}
y \\
x
\end{array}\right)
$$

holds uniformly for $0<x \leq y$ as $y \rightarrow \infty$ when $x$ and $y$ are integers. Moreover if $0<x \leq$ $(\log y)^{1 / 2}$ then for integral $x$ and $y$

$$
s_{x}^{y}=\frac{y !(\log y+\gamma)^{x-1}}{x !}\left\{1+\mathcal{O}\left[(\log y)^{-1 / 2}\right]\right\}
$$

If $(\log y)^{1 / 2}<x \leq y-y^{1 / 3}$ then for real $x$ and $y$

$$
s_{x}^{y}=\frac{\Gamma\left(y+1+u_{1}\right)}{\Gamma\left(u_{1}\right)(2 \pi H)^{1 / 2} u_{1}^{x+1}}[1+\mathcal{O}(1 / x)]
$$


where the $\mathcal{O}$-constant is independent of $x$. If $y-y^{1 / 3} \leq x \leq y$ then independently of $x$ for integral $x$ and $y$

$$
s_{x}^{y}=\left(\begin{array}{l}
y+1 \\
x+1
\end{array}\right)\left(\frac{x}{2}\right)^{y-x}\left[1+\mathcal{O}\left(y^{1 / 3}\right)\right]
$$

THEOREM 3. The function $S_{x}^{y}$ is a log-concave function of $x$ for $\delta<x \leq y$ and $y$ large enough.

REMARK 1. Theorem 3 extends the well-known results that $S_{k}^{n}$ is log-concave as a function of the discrete variable $k$.

REMARK 2. We depend very heavily upon the analysis of Moser- Wyman [6, 7]. We assume the reader has a copy of these papers in hand. The first statement in Theorem 1 and in Theorem 2 is found in Temme [11]. The subsequent statements in each Theorem are derived first following Moser and Wyman. The first statements are then shown to follow. In our approach most of the effort comes from showing that Temme's results are equivalent to the Moser-Wyman results in the extreme values of $x$ with respect to $y$. Since the MoserWyman results are very simply expressed this seems of value in its own right however our main point is that Temme's formula unifies the results of Moser-Wyman. See Section 3 for further discussion.

REMARK 3. It seems very likely that it is not difficult to establish Theorem 2 for real $x$ and $y$ using our definition of $s_{x}^{y}$ and a Theorem 3 for $s_{x}^{y}$ using the Flajolet-Prodinger definition of $s_{x}^{y}$. It would be more work to establish both results using only one definition.

\section{Proofs of the theorems}

\subsection{Proof of Theorem 1}

Let $u_{0}$ be defined as in Theorem 1. A significant difference with the Moser-Wyman analysis arises here. The points $k \pi i$ are just zeros of the integrand when $x$ is an integer and a contour can be moved through them with no difficulty. When $x$ is not an integer we avoid doing so. We deform the contour $C$ to the following contour:Let $C_{1}$ be the straight line $\operatorname{Im}(z)=-2 \pi+\delta, 0<\delta$ however we shall think of $\delta$ as small but fixed and $\operatorname{Re}(z) \leq \epsilon$, where $\epsilon$ is a small positive number. We let $C_{2}$ be the straight line $\operatorname{Re}(z)=\epsilon$, going from 
$\epsilon+i(\delta-2 \pi)$ to the circle $|z|=u_{0}, C_{5}$ and $C_{4}$ be the reflections in the real axis of $C_{1}$ and $C_{2}$ respectively. We let $C_{3}$ be the portion of the circle $|z|=u_{0}$, meeting $C_{2}$ and $C_{4}$. The new contour is $C_{1} \cup C_{2} \cup C_{3} \cup C_{4} \cup C_{5}$ in the counterclockwise sense. We now observe that in the Moser-Wyman analysis [7] $n$ and $k$ can be positive reals. We refer to their analysis leading to their equation (4.3). Note that Stirling's formula gives an asymptotic expansion for $\left(\begin{array}{l}y \\ x\end{array}\right)$ provided $x, y-x \rightarrow \infty$. We obtain from their equation (5.1) (an instance of (4.3), letting $x=m$ and $y=n$ the result

$$
\frac{y !}{x !} \frac{1}{2 \pi i} \int_{C_{3}}\left(e^{z}-1\right)^{x} z^{-y-1} d z=\frac{y !\left(e^{u_{0}}-1\right)^{x}}{x ! 2 u_{0}^{y} \sqrt{\pi x u_{0} H\left(u_{0}\right)}}[1+\mathcal{O}(1 / y)],
$$

where $2 H(u)=e^{u}\left(e^{u}-1\right)^{-1}-u e^{u}\left(e^{u}-1\right)^{-2}$ and $u_{0}$ is denoted by $R$ by Moser and Wyman. This Moser-Wyman result holds uniformly in $x$ if $y-x \geq y^{1 / 3}$. Let us now check that their analysis applies to the generalized Stirling numbers. Note that $u_{0} H\left(u_{0}\right)$ is bounded away from 0 for all $u_{0}$, that $x u_{0} \geq y-x$ by the comment just after the Moser-Wyman equation (3.4c) in [7], and that $C_{3}(R)$ and $C_{4}$ in their equation (5.1) are bounded for all $R$. They do not use the fact that their $m, n$ are integral to derive these facts.

The integrals over $C_{1}, C_{2}, C_{4}$ and $C_{5}$ are easily seen to be $\mathcal{O}\left((2+\epsilon)^{x} /(2 \pi-\delta)^{y}\right)$ since $\left|e^{z}\right|=\exp [\operatorname{Re}(z)] \leq e^{\epsilon}$ and $|z| \geq 2 \pi-\delta$. This error is exponentially small for $\delta<x \leq y$ so our equation (1) holds uniformly in $x$ as $y \rightarrow \infty$ provided $y-x \geq y^{1 / 3}$. We now show that the result stated in Theorem 1 agrees with equation (1). Letting $t_{0}=(y-x) / x$ again and using the Stirling approximation we deduce

$$
\frac{y !}{x !}=\left(\begin{array}{l}
y \\
x
\end{array}\right)(y-x)^{y-x} e^{-(y-x)} \sqrt{2 \pi(y-x)}\left[1+\mathcal{O}\left(y^{-1}\right)\right] .
$$

Thus

$$
\begin{aligned}
e^{A} & =e^{\phi\left(u_{0}\right)-x t_{0}+(y-x) \log t_{0}} \\
& =\frac{\left(e^{u_{0}}-1\right)^{x} e^{-(y-x)} x^{y-x}}{u_{0}^{y}(y-x)^{y-x}}\left[1+\mathcal{O}\left(y^{-1}\right)\right],
\end{aligned}
$$

so

$$
e^{A} x^{y-x} f\left(t_{0}\right)\left(\begin{array}{l}
y \\
x
\end{array}\right)=\frac{y !\left(e^{u_{0}}-1\right)^{x}}{x ! u_{0}^{y}} \frac{f\left(t_{0}\right)}{\sqrt{2 \pi(y-x)}}\left[1+\mathcal{O}\left(y^{-1}\right)\right] .
$$

Now

$$
f\left(t_{0}\right)=\left[(y-x) / u_{0}^{2} \phi^{\prime \prime}\left(u_{0}\right)\right]^{1 / 2}, \quad \phi^{\prime \prime}\left(u_{0}\right)=y u_{0}^{-2}-x e^{u_{0}}\left(e^{u_{0}}-1\right)^{-2}
$$

and using $y / x=e^{u_{0}} u_{0} /\left(e^{u_{0}}-1\right)$ we find that

$$
\begin{aligned}
u_{0}^{2} \phi^{\prime \prime}\left(u_{0}\right) & =x\left[\frac{y}{x}-\frac{u_{0} e^{u_{0}}}{\left(e^{u_{0}}-1\right)^{2}}\right] \\
& =x u_{0}\left[\frac{e^{u_{0}}}{e^{u_{0}}-1}-\frac{u_{0} e^{u_{0}}}{\left(e^{u_{0}}-1\right)^{2}}\right]=2 x u_{0} H\left(u_{0}\right),
\end{aligned}
$$


so

$$
e^{A} x^{y-x} f\left(t_{0}\right)\left(\begin{array}{l}
y \\
x
\end{array}\right)=\frac{y !\left(e^{x}-1\right)^{x}}{x ! 2 u_{0}^{y} \sqrt{\pi u_{0} H\left(u_{0}\right)}}\left[1+\mathcal{O}\left(y^{-1}\right)\right] .
$$

Thus if $y-x \geq y^{1 / 3}$ the first result of Theorem 1 follows from the Moser-Wyman results.

We turn to the case $y-x<y^{1 / 3}$ and again follow Moser-Wyman starting at their equation (2.1) and continuing to equation (2.11). We write with Flajolet-Prodinger

$$
S_{y-x}^{y}=\frac{y !}{(y-x) ! 2 \pi i} \int_{C}\left(\frac{e^{z}-1}{z}\right)^{y-x} z^{-x-1} d z .
$$

If one uses

$$
\log \frac{e^{z}-1}{z}=\frac{z}{2}+\sum_{k=1}^{\infty}(-1)^{k} \frac{\zeta(2 k)}{k(2 \pi)^{2 k}} z^{2 k},
$$

and the substitutions $z=2 w /(y-x), q=2 /(y-x)$ one obtains, as do Moser and Wyman,

$$
\begin{aligned}
\left(\frac{e^{z}-1}{z}\right)^{y-x} & =\exp \left[w+\sum_{k=1}^{\infty}(-1)^{k+1} \frac{2 \zeta(2 k) w^{2 k}}{k(2 \pi)^{2 k}} q^{2 k-1}\right] \\
& =e^{w} \sum_{k=0}^{\infty} P_{k}(w) q^{k}
\end{aligned}
$$

where the $P_{k}(w)$ are polynomials in $w$ with degree at least $k+1$ and at most $2 k$. Thus agreeing with Moser and Wyman we get

$$
S_{y-x}^{y}=\left(\begin{array}{l}
y \\
x
\end{array}\right) \frac{x ! q^{-x}}{2 \pi i} \int_{C} e^{w} w^{-x-1}\left[1+\frac{w^{2} q}{12}+\frac{w^{4} q^{2}}{288}+\left(\frac{w^{6}}{10,368}-\frac{w^{4}}{1440}\right) q^{3}+\cdots\right] d w
$$

Now unlike Moser and Wyman, we use the classical identity, due to Hankel

$$
\frac{1}{2 \pi i} \int_{C} e^{w} w^{-t-1} d w=\frac{1}{\Gamma(t)}
$$

to obtain

$$
\begin{aligned}
S_{y-x}^{y} & =\left(\begin{array}{l}
y \\
x
\end{array}\right) q^{-x} x !\left[\frac{1}{x !}+\frac{q}{12(x-2) !}+\frac{q^{2}}{288(x-4) !}+\cdots\right] \\
& =\left(\begin{array}{l}
y \\
x
\end{array}\right) q^{-x}\left[1+\frac{(x)_{2}}{6(y-x)}+\frac{(x)_{4}}{72(y-x)^{2}}+\left(\frac{(x)_{6}}{1296}-\frac{(x)_{4}}{180}\right) /(y-x)^{3}+\cdots\right]
\end{aligned}
$$

For the reasons that Moser and Wyman give, this is not only an asymptotic series it is a convergent series for $x=o\left((y-x)^{1 / 2}\right)$. We therefore conclude that

$$
S_{y-x}^{y}=\left(\begin{array}{l}
y \\
x
\end{array}\right)\left(\frac{y-x}{2}\right)^{x}\left[1+\mathcal{O}\left(y^{-1 / 3}\right)\right]=\frac{y^{2 x}}{2^{x} x !}\left[1+\mathcal{O}\left(y^{-1 / 3}\right)\right]
$$

if $y-x=\mathcal{O}\left(x^{1 / 3}\right)=\mathcal{O}\left(y^{1 / 3}\right)$. 
REMARK 1. This analysis can be replaced by the general technique known as Watson's Lemma for loop integrals see Olver [9].

We now show that this completes the proof of Theorem 1. We start with

$$
\phi\left(u_{0}\right)=-(y-x) \log u_{0}+x \log \left[\left(e^{u_{0}}-1\right) / u_{0}\right]
$$

and

$$
\frac{e^{u_{0}}-1}{u_{0}}=\frac{1-e^{-u_{0}}}{e^{-u_{0}} u_{0}}=\frac{x}{y} e^{u_{0}},
$$

so

$$
\log \left(\frac{e^{u_{0}}-1}{u_{0}}\right)=\log (x / y)+u_{0}
$$

Thus

$$
\phi\left(u_{0}\right)=-(y-x) \log u_{0}+x u_{0}+x \log [1-(y-x) / y] .
$$

Now

$$
1-(y-x) / y=x / y=\left(1-e^{-u_{0}}\right) / u_{0}=1-u_{0} / 2+u_{0}^{2} / 6+\mathcal{O}\left(u_{0}^{3}\right)
$$

implies

$$
u_{0}=\frac{2(y-x)}{y}\left\{1+\frac{4(y-x)^{2}}{3 y^{2}}+\mathcal{O}\left[(1-x / y)^{3}\right]\right\}
$$

so

$$
\phi\left(u_{0}\right)=-(y-x) \log u_{0}+y-x+\mathcal{O}\left[(y-x)^{2} / y\right] .
$$

Also

$$
A=\phi\left(u_{0}\right)-x t_{0}+(y-x) \log t_{0}=(y-x) \log (y-x)-(y-x) \log u_{0}-(y-x) \log x .
$$

From (3)

$$
(y-x) \log u_{0}=(y-x) \log 2+(y-x) \log (y-x)-(y-x) \log y+\mathcal{O}\left[(y-x)^{3} / y^{2}\right],
$$

so

$$
\begin{aligned}
A & =-(y-x) \log 2+(y-x) \log (y / x)+\mathcal{O}\left[(y-x)^{2} / y\right] \\
& =-(y-x) \log 2+\mathcal{O}\left[(y-x)^{2} / y\right] .
\end{aligned}
$$

Thus

$$
e^{A} x^{y-x} f\left(t_{0}\right)\left(\begin{array}{l}
y \\
x
\end{array}\right)\left[1+\mathcal{O}\left(y^{-1 / 3}\right)\right]=\frac{y^{2(y-x)} f\left(t_{0}\right)}{2^{y-x}(y-x) !}\left[1+\mathcal{O}\left(y^{-1 / 3}\right)\right]
$$


if $y-x=\mathcal{O}\left(y^{1 / 3}\right)$. Now $f\left(t_{0}\right)=\left[t_{0} /\left(1+t_{0}\right)\left(u_{0}-t_{0}\right)\right]^{1 / 2}$ and $t_{0} /\left(1+t_{0}\right)=(y-x) / y$. Furthermore $u_{0}-t_{0}=2(y-x) y^{-1}+\mathcal{O}\left[(y-x)^{2} / y^{2}\right]-(y-x) / x=(y-x) / y+\mathcal{O}\left[(y-x)^{2} / y\right]$, so $f\left(t_{0}\right)=1+\mathcal{O}\left[(y-x)^{2} / y\right]$. Thus if $y-x=\mathcal{O}\left(y^{1 / 3}\right)$ the first statement of Theorem 1 is equivalent to

$$
\frac{y^{2(y-x)}}{2^{y-x}(y-x) !}\left[1+\mathcal{O}\left(y^{-1 / 3}\right)\right]
$$

which agrees with equation (2); note that the $x$ of equation (2) is $y-x$ here.

This proves Theorem 1.

\subsection{Proof of Theorem 2}

If $h(n) \leq m \leq n-n^{a}$, where $h(n)$ is any function tending to infinity with $n$, and $a$ is a positive constant $<1 / 2$ then Moser-Wyman [6] show that (they denote $u_{1}$ by $R$ )

$$
\begin{aligned}
s_{m+1}^{n+1} & =\frac{\Gamma\left(1+n+u_{1}\right)}{\left[2 \pi H\left(u_{1}\right)\right]^{1 / 2} u_{1}^{m+1} \Gamma\left(u_{1}\right)}[1+\mathcal{O}(1 / m)] \\
& =\frac{\left(n+u_{1}\right) !}{\left[2 \pi H\left(u_{1}\right)\right]^{1 / 2} u_{1}^{m} u_{1} !}[1+\mathcal{O}(1 / m)],
\end{aligned}
$$

where

$$
H=m-\sum_{k=1}^{n} u_{1}^{2} /\left(u_{1}+k\right)^{2} .
$$

The fact that $m$ and $n$ are integers is not essential in their analysis. We can choose the contour of integration as they do. We let

$$
\begin{aligned}
\psi(u) & =\log \Gamma(u+y+1)-\log \Gamma(u+1)-x \log u, \\
\psi^{\prime}(u) & =\Psi(u+y+1)-\Psi(u+1)-x / u, \\
\Psi(u) & =\frac{d}{d u} \Gamma(u),(\text { our } \Psi \text { is Moser }- \text { Wyman's } \psi),
\end{aligned}
$$

etc., and use the asymptotic estimates for these functions given in Chapter 6 of [1]. One then obtains the same asymptotic estimates as if $x$ and $y$ were integers but the discrete sums we write should be interpreted in terms of $\psi$ and its derivative. With this interpretation we get

$$
e^{\psi\left(u_{1}\right)}=\frac{\left(u_{1}+y\right) !}{u_{1}^{x} u_{1} !}[1+\mathcal{O}(1 / m)]
$$

Also

$$
u_{1}^{2}=x-\frac{u_{1}^{2}}{\left(u_{1}+1\right)^{2}}-\cdots-\frac{u_{1}^{2}}{\left(u_{1}+y\right)^{2}}=H
$$

Thus

$$
g\left(t_{1}\right)=\frac{1}{u_{1} \sqrt{H}} \sqrt{x(y-x) / y} .
$$


Furthermore

$$
\left(t_{1}+1\right)^{-y}=\left(\frac{y}{y-x}\right)^{-y}=\frac{(y-x)^{y}}{y^{y}}, \quad t_{1}^{x}=\frac{x^{x}}{(y-x)^{x}}
$$

so, using Stirling's approximation,

$$
e^{B} g\left(t_{1}\right)\left(\begin{array}{l}
y \\
x
\end{array}\right)=\frac{\Gamma\left(y+u_{1}+1\right)}{\sqrt{2 \pi H} u_{1}^{x+1}}[1+\mathcal{O}(1 / x)]
$$

which agrees with the Moser-Wyman result.

Suppose now that $x=\mathcal{O}\left(\log ^{1 / 2} y\right)$. Then $t_{1}=x y^{-1}(1-x / y)^{-1}=x / y+\mathcal{O}\left(\log y / y^{2}\right)$. To solve for $u_{1}$ note that

$$
\psi\left(u_{1}\right)=\frac{1}{1+u_{1}}+\frac{1}{2+u_{1}}+\cdots+\frac{1}{y+u_{1}}=\log \left(u_{1}+y\right)+\gamma+\mathcal{O}(1 / y)
$$

(even when $y$ is not an integer), so

$$
u_{1} \log \left(u_{1}+y\right)=\frac{x}{\log y+\gamma}+\mathcal{O}(1 / y)=\mathcal{O}\left(1 / \log ^{1 / 2} y\right) .
$$

Now

$$
\begin{aligned}
e^{\psi\left(u_{1}\right)} & =\frac{\Gamma\left(u_{1}+y+1\right)}{\Gamma\left(u_{1}\right)}=y ! u_{1}^{-x}\left[1+\mathcal{O}\left(\log ^{-1 / 2} y\right)\right] \\
& =y !(\log y+\gamma)^{x} x^{-x}\left[1+\mathcal{O}\left(\log ^{-1 / 2} y\right)\right]
\end{aligned}
$$

Furthermore

$$
\begin{gathered}
\left(1+t_{1}\right)^{-y}=(1-x / y)^{y}=e^{-x}\left[1+\mathcal{O}\left(y^{-1} \log y\right)\right], \\
t_{1}^{x}=\frac{x^{x}}{(y-x)^{x}}=\frac{x^{x} e^{x}}{y^{x}}\left[1+\mathcal{O}\left(y^{-1} \log y\right)\right],
\end{gathered}
$$

so, using $y ! /\left((y-x) ! \sim y^{x}\left[1+\mathcal{O}\left(y^{-1} \log y\right)\right]\right.$, we have

$$
e^{B}\left(\begin{array}{l}
y \\
x
\end{array}\right) g\left(t_{1}\right)=\frac{y !(\log y+\gamma)^{x} g(t)}{x !}\left[1+\mathcal{O}\left(\log ^{-1 / 2} y\right)\right] .
$$

Finally, if $x$ is bounded away from 0 ,

$$
u_{1}^{2} \psi^{\prime \prime}\left(u_{1}\right)=x+\mathcal{O}\left(u_{1}^{2}\right)=x+\mathcal{O}\left(\log ^{-1} y\right),
$$

so

$$
g\left(t_{1}\right)=\frac{x}{x+\mathcal{O}\left(\log ^{-1} y\right)}=1+\mathcal{O}\left(\log ^{-1} y\right)
$$

thus

$$
e^{B} g\left(t_{1}\right)\left(\begin{array}{l}
y \\
x
\end{array}\right)=\frac{y !(\log y+\gamma)^{x}}{x !}\left[1+\mathcal{O}\left(\log ^{-1} y\right)\right],
$$

which agrees with Moser and Wyman if $x=\mathcal{O}\left(\log ^{1 / 2} y\right)$ when $x$ and $y$ are integral. 
We now consider the remaining range $y-x=\mathcal{O}\left(y^{a}\right)$, where $0<a$ is a constant $<1 / 2$. We note first of all that we can follow Moser-Wyman making only very minor changes to get (choosing their $a=1 / 3$ ) to show that when $x$ and $y$ are integral

$$
s_{x+1}^{y+1}=\left(\begin{array}{l}
y+1 \\
x+1
\end{array}\right)(x / 2)^{y-x}\left[1+\mathcal{O}\left(y^{-1 / 3}\right)\right] .
$$

In fact, Moser and Wyman give a convergent series for $s_{x+1}^{y+1}$, not only the first term, which is a complete asymptotic expansion.

We now show that the first expression in Theorem 2 (which is Temme's) agrees with this formula. We begin by deriving an estimate for $u_{1}$. Note that

$$
\psi^{\prime}\left(u_{1}\right)=\frac{1}{1+u_{1}}+\frac{1}{2+u_{1}}+\cdots+\frac{1}{y+u_{1}}-x / u_{1}
$$

and that the above sum can be estimated by Euler-Maclaurin summation in a routine way, giving

$$
\log \left(1+y / u_{1}\right)+\mathcal{O}\left(1 / u_{1}\right)=x / u_{1}
$$

or

Thus

$$
\left.\frac{y}{u_{1}}-\frac{y^{2}}{2 u_{1}^{2}}+\mathcal{O}\left[\left(y / u_{1}\right)^{3}\right)\right]=\frac{x}{u_{1}} .
$$

$$
\begin{aligned}
u_{1} & =\frac{y^{2}}{2(y-x)}+\mathcal{O}\left[\frac{y^{3}}{u_{1}(y-x)}\right]=\frac{y^{2}}{2(y-x)}+\mathcal{O}(y) \\
& =\frac{y^{2}}{2(y-x)}\left[1+\mathcal{O}\left(\frac{y-x}{y}\right)\right]
\end{aligned}
$$

Now

$$
e^{\psi\left(u_{1}\right)}=\left(u_{1}+y\right) ! e^{-y} u_{1}^{-x} / u_{1} !
$$

and from Stirling's approximation this is

$$
\begin{aligned}
e^{\psi\left(u_{1}\right)} & =\left(1+y / u_{1}\right)^{u_{1}}\left(1+y / u_{1}\right)^{y} e^{-y} u_{1}^{y-x}[1+\mathcal{O}(1 / y)] \\
& \left.=e^{y-y^{2} / 2 u_{1}+\mathcal{O}\left(y^{3} / u_{1}^{2}\right)} \frac{y^{2(y-x)}}{2(y-x)^{y-x}}\left\{1+\mathcal{O}\left[(y-x)^{2} / y\right)\right]\right\} \\
& \left.=\frac{y^{2 y-2 x} e^{y-x}}{2^{y-x}(y-x)^{y-x}}\left\{1+\mathcal{O}\left[(y-x)^{2} / y\right)\right]\right\} .
\end{aligned}
$$

Furthermore

$$
\left(1+t_{1}\right)^{y}=y^{y} /(y-x)^{y}, \quad t_{1}^{x}=x^{x} /(y-x)^{x},
$$

so

$$
e^{\psi\left(u_{1}\right)}\left(1+t_{1}\right)^{-y} t_{1}^{x}=\frac{e^{y-x}}{2^{y-x}} y^{y-x}\left(\frac{x}{y}\right)^{x}
$$


Since

we have that

$$
\left.\left(\frac{x}{y}\right)^{x}=\left(\frac{x}{x+y-x}\right)^{x}=e^{x-y}\{1+\mathcal{O}[(y-x) / y)]\right\}
$$

$$
\begin{aligned}
e^{\psi\left(u_{1}\right)}\left(1+t_{1}\right)^{-y} t_{1}^{x} & \left.=\left(\frac{y}{2}\right)^{y-x}\left\{1+\mathcal{O}\left[(y-x)^{2} / y\right)\right]\right\} \\
& \left.=\left(\frac{x}{2}\right)^{y-x}\left\{1+\mathcal{O}\left[(y-x)^{2} / y\right)\right]\right\}
\end{aligned}
$$

Theorem 2 will now be proved if we show that $g\left(t_{1}\right) \sim 1$. Note

$$
\begin{aligned}
\left.u_{1}^{2} \psi^{\prime \prime} u_{1}\right) & =-\frac{u_{1}^{2}}{\left(u_{1}+1\right)^{2}}-\cdots-\frac{u_{1}^{2}}{\left(u_{1}+y\right)^{2}}+x \\
& =-\frac{1}{\left(1+u_{1}^{-1}\right)^{2}}-\cdots-\frac{1}{\left(1+y u_{1}^{-1}\right)^{2}}+x \\
& =-y+\frac{y^{2}}{u_{1}}+\mathcal{O}\left[(y-x)^{2} / y^{2}\right]+x \\
& =-y+2(y-x)+\mathcal{O}\left((y-x)^{3} / y^{2}\right)+x=y-x+\mathcal{O}\left[(y-x)^{3} / y^{2}\right] .
\end{aligned}
$$

Since

$$
g\left(t_{1}\right)=\frac{1}{u_{1}} \sqrt{\frac{x(y-x)}{y \psi^{\prime \prime}\left(u_{1}\right)}}, \quad y=x\left[1+\mathcal{O}\left(y^{-1 / 3}\right)\right]
$$

we have $g\left(t_{1}\right)=1+\mathcal{O}\left(y^{-1 / 3}\right)$ and Theorem 2 is proved.

\subsection{Proof of Theorem 3}

We follow the approach of Richmond-Merlini [10], however we use the Moser-Wyman results in place of Gardy's results [3]. The notation below is that of Moser- Wyman and Gardy. (Gardy's Theorem 3 was designed to hold under as general a class of functions as possible not only for the Stirling numbers). Clearly $x u_{0}<y$ and equation (3.4) of Moser- Wyman [7] shows $x u_{0}>y-x$ so $x u_{0}$ plays the role of $y$ in Gardy's work and also in that of RichmondMerlini. Gardy's $\left.\delta h(\rho)=\rho /\left(1-e^{-\rho}\right)\right)$ is also the coefficient of $\theta^{2}$ in Moser-Wyman's $g(\theta, R)$. Moser-Wyman show that the coefficients, $c_{k}$, of $\theta^{k}$ satisfy $c_{k} \leq M R, M$ an absolute constant. The significant range of integration in Moser-Wyman is $|\theta| \leq(m R)^{-3 / 8}=(m R)^{1 / 8}(m R)^{-1 / 2}$, so the factor $\log y$ in Gardy's work is replaced by $(m R)^{1 / 8}$ or $y^{1 / 8}$. This does not matter since Gardy's $h(\rho)=\exp (\rho)-1$ and

$$
\theta^{k} c_{k}=\left.\theta^{k} \frac{d^{k} \log h\left(\rho e^{i \theta}\right)}{d^{k} \theta}\right|_{\theta=0}=\mathcal{O}\left[(m R)^{1-3 k / 8}\right]
$$

and so the terms with $k \geq 3$ are negligible. The $\mathcal{O}\left(y^{-1} \log ^{2} y\right)$ terms in Merlini-Richmond become $\mathcal{O}\left(y^{-3 / 4}\right)$ using the Moser-Wyman analysis. Thus corresponding to Theorem 3.6 of 
[10] we have: If $y-x \geq y^{1 / 3}$, then

$$
\begin{aligned}
\frac{d S_{x}^{y}}{d x} & \sim S_{x}^{y}[\log h(\rho)-\log x] \\
\frac{d^{2} \log S_{x}^{y}}{d^{2} x} & \sim S_{x}^{y}\left[-\log ^{2} x-\frac{1}{x}+\frac{h^{\prime}(\rho)}{h(\rho)} \frac{d \rho}{d x}\right] .
\end{aligned}
$$

Furthermore, Corollary 3.9 of Richmond-Merlini[10] holds; it says that the term involving $d \rho / d x$ is $<0$, so $S_{x}^{y}$ is log-concave for $y-x \geq y^{1 / 3}$.

If $y-x=\mathcal{O}\left(y^{1 / 3}\right)$ we can use the exact expression for $S_{x}^{y}$ obtained in the proof of Theorem 1. It follows that

$$
\log S_{x}^{y}=\log (y+1) !-\log (x+1) !-\log (y-x) !+(y-x) \log (x / 2)+\cdots,
$$

and since the convergent series and the Stirling asymptotic series for the factorial function can be differentiated term by term, we get

$$
\frac{d^{2} \log S_{x}^{y}}{d^{2} x}=-\frac{1}{y-x}+\mathcal{O}\left(y^{-2 / 3}\right)
$$

So again $S_{x}^{y}$ is $\log$-concave.

\section{Closing Comments}

It is clear that while we have derived Temme's results in the slightly more general context of generalized Stirling numbers, the situation is not satisfactory. It would be much preferable to show how to choose the contour of integration so that Temme's $f(t)$ (or $G(t)$ ) has no singularities on or in the contour of integration, indeed to see how to do this for integrals other than those representing the Stirling numbers. The Moser-Wyman technique for the extreme ranges can be replaced by Watson's Lemma for loop integrals or other quite general methods.

The log-concavity results show that the generalized Stirling numbers of the second kind have a unique maximum for $\delta<x \leq y$ for large $y$. The problem of showing this for integral variables seems to be still open. Erdös [2] has shown that the Stirling numbers of the first kind have a unique maximum when the variables are integral. 


\section{References}

[1] M. Abramowitz, M., Stegun, I.A.: Handbook of Mathematical Functions with Formulas, Graphs and Mathematics Tables, National Bureau of Standards, Applied Series 55, U.S. Government Printing Office, Washington, D.C. (1964).

[2] Erdös, P.: On a Conjecture of Hammersley, J. Math. Soc., 28, 232-236, (1953).

[3] Gardy, D.: Some results on the asymptotic behaviour of coefficients of large powers of functions, Discrete Math., 139, 189-217, (1995).

[4] Flajolet, P., Prodinger, H.: On Stirling Numbers for Complex Arguments and Hankel Contours, SIAM J. of Discrete Math. 12, 155-159, (1999).

[5] Graham, R.L., Knuth, D.E., Patashnik, O.: Concrete Mathematics, second edition, Addison Wesley (1994).

[6] Moser, L., Wyman, M.: Asymptotic development of the Stirling numbers of the first kind, J. London Math. Soc. 33, 133-146, (1958).

[7] Moser, L., Wyman, M.: Stirling numbers of the second kind, Duke Math. J. 25, 29-43, (1958).

[8] Odlyzko, A.M.: Asymptotic methods in enumeration, in Handbook of Combinatorics, Vol. 2, R. Graham, M. Grötschel and L. Lóvasz, Eds., Elsevier (1995).

[9] Olver, F. W. J.: Asymptotics and Special Functions, A. K. Peters (1997).

[10] Richmond, B., Merlini, D.: Stirling numbers for complex arguments, SIAM J. of Discrete Math., 10, 73-82, (1997).

[11] Temme, N.M.: Asymptotic estimates of Stirling numbers, Studies in Applied Math. 89, 233-243, (1993).

R. Chelluri, Department of Mathematics, Cornell University, Ithaca, New York 14850, U.S.A. e-mail tc39@cornell.edu

L.B. Richmond, Department of Combinatorics and Optimization, University of Waterloo, Waterloo, Ontario, N2L 3G1, Canada

e-mail lbrichmo@watdragon. uwaterloo.ca

N.M. Temme, C.W.I., PO. Box 94079, 1090 GB Amsterdam, The Netherlands

e-mail nicot@cwi.nl 